NASH and 7\% Hepatitis C. Median MELD score was 15.5 (IQR 13.5-21). All patients were prescribed lactulose. Of the 30 patients, 5 died within 6 months of commencing Rifaximin. One patient was discontinued, due to non-compliance. 24 patients were included in the final analysis. We compared the outcomes for the 6 months prior to, and the 6 months after commencing Rifaximin treatment. Median hospital admissions were reduced from 2 admissions (IQR 1-3, Range 1-5) to 1 admission (IQR $0-2$, Range $0-4$, Wilcoxon $\mathrm{p}<0.05$ ). Median number of bed days was reduced from 27.5 (IQR 16.0-35.3, Range 2-129) to 2.5 (IQR 0-23.5, Range 0-55, Wilcoxon $\mathrm{p}<0.05$ ). No patient developed Clostridium difficile-associated diarrhoea in the 6 months after commencing Rifaximin.

Conclusion In our hospital, the basic cost of a hospital bed day is $£ 300$. A 6 month course of Rifaximin costs $£ 1688$. This study demonstrates that Rifaximin treatment in patients with HE, due to chronic liver disease, produced a marked reduction in hospital admissions and hospital bed day occupancy in a District General Hospital, with major cost savings and improved clinical outcomes.

Disclosure of Interest None Declared.

\section{PWE-126 SPONTANEOUS BACTERIAL PERITONITIS (SBP) IS RARE AMONG ROUTINE ELECTIVE DAY-CASE THERAPEUTIC PARACENTESIS: IS ROUTINE FLUID ANALYSIS JUSTIFIED?}

A Sugumaran*, D Martin, M Czajkowski, A Yeoman. Gwent Liver Unit, Newport, UK

10.1136/gutjnl-2014-307263.386

Introduction SBP is a serious infection in patients with cirrhosis and is associated with a high risk of mortality. EASL guidelines recommend a diagnostic tap and testing of white cell count on samples for all patients with new onset ascites or those hospitalised with deterioration. Whether this is still necessary in patients undergoing elective paracentesis for diuretic resistant ascites is unknown. We set out to determine the incidence of SBP in a cohort of patients undergoing ascitic tap on an inpatient or elective outpatient basis.

Methods We retrospectively analysed data from the Gwent Liver Unit database and compared rates of SBP between those having an ascitic tap during an acute admission and those who underwent paracentesis as an elective day-case. SBP was confirmed by the presence of $>250$ polymorphs $/ \mathrm{ml}^{3}$.

Results In total 274 ascitic tap samples were reviewed, 176 from inpatients and 98 following elective paracentesis.

22 episodes of SBP were diagnosed (total incidence 8\%).

21 episodes of SBP occurred among inpatients (incidence $12 \%)$ and just 1 in elective paracentesis patients (1\%). However this individual had a previous SBP but unfortunately did not continue on her secondary antibiotic prophylaxis. Organisms were identified in 9 of 21 (42\%) inpatients with SBP (3 E coli, 3 Klebsiella and 3 Streptococci) but none was identified in the only elective outpatient with SBP.

Conclusion The incidence of SBP is extremely low among elective outpatients so the recommendation to routinely test for SBP in this cohort is questionable unless clinical features suggest an acute deterioration.

Disclosure of Interest None Declared.

\section{PWE-127 TRENDS IN HOSPITAL ADMISSIONS WITH ALCOHOL} RELATED LIVER DISEASE IN SOUTH-EAST ENGLAND

${ }^{1} \mathrm{~N}$ Shah, ${ }^{1} \mathrm{BD}$ Warner*, ${ }^{1} \mathrm{~J}$ Potts, ${ }^{2} \mathrm{~S}$ Verma. 'Department of Gastroenterology, Royal Sussex County Hospital, UK; ${ }^{2}$ Department of Gastroenterology, Royal Sussex Hospital, Brighton, UK

\subsection{6/gutjnl-2014-307263.387}

Introduction Morbidity and mortality from alcohol related liver disease (ALD) has increased significantly in England. We aimed to assess the impact of the change in the alcohol licensing law in 2005 on hospital admission as well as morbidity and mortality from ALD.

Methods This was a retrospective study between Jan 2006 to Dec 2011. Patients were identified using the ALD code (K70). Our institute is a teaching hospital in South-East England with a referral base of $\sim 300,000$ patients. Biochemical, radiological and microbiology data were collected for each patient from computerised records. The reason for hospital presentation, including the length-of-stay (and ITU stay) were collected. The data was analysed using the $t$ test.

Results During the study period there were a total of 294 admissions due to ALD. Amongst these, 33\% presented to hospital with one admission and $67 \%$ had more than 1 admission. 69\% were men (201/294), 31\%(93/294) were women. The median age of the women and men in 2006 and 2007 was 56 and 52 years respectively. However, between 2008-2011, the median age of women presenting was 49 years. There was a rise in the number of admissions related to ALD each year: $40 \%$ $(116 / 294)$ in $2006,45 \%(133 / 294)$ in $2007,59 \%(172 / 294)$ in $2008,59 \%(171 / 294)$ in $2009,66 \%(193 / 294)$ in 2010 and $68 \%$ $(201 / 294)$ in 2011. Presentation with hyperbilirubinaemia was the most common cause of hospital admission at $72 \%(211 /$ 294), followed by gastrointestinal bleeding at 60\%(179/294). Spontaneous bacterial peritonitis contributed to $22 \%(66 / 294)$ of admissions. $17 \%(51 / 294)$ had gram-negative and 6\%(17/294) presented with gram-positive sepsis, whereas 15\%(46/294) had infection of unknown origin. 28\%(82/294) warranted ITU admission for organ support. The overall in-hospital mortality was 33\%(96/294). The MELD in patients who died was $25 \pm$ 0.9 vs $16.94 \pm 0.2$ in those that survived $(\mathrm{p}<0.0001)$ each year. The percentage of failing organs each year is shown in the table below.

Conclusion A rise in the number of admissions and re-admissions with ALD in particular with acute on chronic liver failure is a major burden on health services. It is associated with high mortality and with multi-organ failure. Increasing awareness of alcohol as a health hazard needs to be a public health priority. Early recognition of acute on chronic liver failure and timely intervention to prevent its progression is required.

Disclosure of Interest None Declared.

\begin{tabular}{lllll}
\multicolumn{6}{l}{ Abstract PWE-127 Table 1 } \\
\hline & 3 organs & 2 organs & 1 organ & none \\
\hline 2006 & $13 / 120(10 \%)$ & $9 / 120(7.5 \%)$ & $83 / 12069 \%)$ & $15 / 120(12.5 \%)$ \\
2007 & $15 / 125(12 \%)$ & $15 / 125(12 \%)$ & $73 / 125(58.4 \%)$ & $22 / 125(18.3 \%)$ \\
2008 & & & & \\
2009 & $12 / 131(9.2 \%)$ & $24 / 131(18 \%)$ & $79 / 131(60 \%)$ & $15 / 131(11.5 \%)$ \\
2010 & & & & \\
2011 & $9 / 114(8 \%)$ & $19 / 114(17 \%)$ & $64 / 114(56 \%)$ & $22 / 114(19 \%)$ \\
\hline
\end{tabular}

